Chapter 8

\title{
Intestinal Fibroblast/Myofibroblast TRP Channels in Inflammatory Bowel Disease
}

\author{
Lin Hai Kurahara, Keizo Hiraishi, Kunihiko Aoyagi, \\ Yaopeng Hu, Miho Sumiyoshi and Ryuji Inoue
}

Additional information is available at the end of the chapter

http://dx.doi.org/10.5772/64830

\begin{abstract}
Inflammatory bowel disease (IBD) is characterized by the repeated cycles of inflammation and healing of the gut, which ultimately progress into intestinal fibrosis. Colonic fibroblast/myofibroblast's functions such as transformation, proliferation, invasion, migration, stress fiber formation, collagen synthesis, and cytokine/chemokine secretion are well estimated. However, the detailed mechanism can rarely be found so far. Thus, we focused on transient receptor potential (TRP) protein super family activated by various physical/chemical stimulations based on the above-described recognitions and also conducted the following examinations for the potential roles in $\mathrm{Ca}^{2+}$ signal transduction in fibroblast/myofibroblasts cells, which play an important role in intestinal inflammation and tissue remodeling. This chapter not only facilitates the understanding about the new role of intestinal fibroblast/myofibroblasts TRP channel for regulating inflammation, fibrotic processes but also suggests a novel molecular target of IBD treatment in future.
\end{abstract}

Keywords: inflammatory bowel disease (IBD), myofibroblast, TRP channels

\section{Introduction}

The prevalence of inflammatory bowel disease (IBD), a group of idiopathic disorders such as Crohn's disease (CD) and ulcerative colitis (UC) that cause chronic inflammation or ulcers in large- and small-intestinal mucosa, has been rapidly increasing since the Second World War. Because IBD follows a course of repeated severe diarrhea and constipation from a young age, it deteriorates an individual's quality of life for a long period of time as a refractory disorder. 
Currently, most IBD treatments are limited to symptomatic relief. With increasing incidence, there is an escalating need to clarify a cause and establish definitive treatments $[1,2]$.

Located at the interface between the epithelium and lamina propria in most mucosal tissues, intestinal fibroblast/myofibroblast cells have ultrastructural features reminiscent of both smooth muscle cells and fibroblasts. Accumulating evidence suggests that myofibroblasts play crucial roles in intestinal homeostasis, inflammation, and neoplasia. In addition, these cells are known to play an essential role in modulating wound healing and fibrosis processes at the time of tissue damage or inflammation [3-5]. For instance, during skin-wound healing, fibroblast cells differentiate into myofibroblasts that secrete cytokines and growth factors to reduce wound size by contracting granulation tissue. Similarly, fibroblast-derived hepatic stellate cells (also known as Ito cells) located in the sinusoidal space of the liver support sinusoidal structure. Fibroblasts with similar transformation ability are also distributed in renal tubular epithelia, where they can be transformed in response to tissue damage, inflammatory substances, or growth factors to promote collagen production and stress fiber formation for tissue fibrosis [6-8]. Furthermore, fibroblast/myofibroblast cells produce cytokines, chemokines, growth factors, and inflammatory mediators involved in immune and inflammatory responses. The activation of myofibroblasts can induce excessive fibrosis, causing pathological tissue modifications (remodeling) such as wound closure, keloid formations, hepatic fibrosis (cirrhosis), and digestive tract obstructions [9]. However, mechanisms underlying myofibroblast transformation and cytokine secretion remain almost completely unknown, despite their importance in inflammatory tissue modifications.

Fibroblasts/myofibroblasts play important roles during the processes of intestinal inflammation and tissue remodeling [10, 11]; however, detailed mechanisms have rarely been identified. Based on previously described recognitions, we therefore focused on the transient receptor potential (TRP) superfamily as a new $\mathrm{Ca}^{2+}$ channel gene group activated by various physical and chemical stimuli. Mammalian TRP proteins form a non-selective cation channel superfamily that includes approximately 30 isoforms categorized into six subfamilies [12], including TRPC (canonical or classical: TRPC1-7), TRPV (vanilloid: TRPV1-6), TRPM (melastatin: TRPM1-8), TRPP (polycystin: TRPP1-4), TRPML (mucolipin: TRPML1-3), and TRPA (ankyrin: TRPA1). Implicated in a variety of cellular functions, TRP proteins form large non-voltagegated cation channels constitutively activated by various physicochemical stimuli. Known activators for TRP channels include chemical stimuli (such as receptor stimulation, change in $\mathrm{pH}$, and spicy or cooling agents), as well as temperature changes and various forms of mechanical stimuli including osmotic stress, membrane stretching, and shear forces. Proposed mechanisms are primarily associated with lipid bilayer mechanics, specialized force-transducing structures, biochemical reactions, membrane trafficking, and transcriptional regulation. TRP channels are assumed to form a tetrameric structure with four homologous subunits consisting of a six transmembrane segments, S1-S6, which are flanked by N- and C-terminal cytosolic regions. Although the six-time membrane-spanning configuration and a short helical pore loop between S5 and S6 segments are the hallmarks of voltage-gated cation channels, in TRP channels, periodically arranged, positively charged amino acid residues in the S4, which are essential for voltage-sensing, are missing [13]. Further, many additional protein-to-protein 
interaction domains and phosphorylation motifs exist within the $\mathrm{N}$ - and C-terminals of TRP channels. It is believed that, within specific membrane domains (e.g., caveola), a variety of signaling complexes are formed through these interaction sites, wherefrom diverse intracellular signal transductions are initiated. Owing to ubiquitous expression over the whole body including the central/peripheral nerve, cardiovascular, respiratory, digestive, renal urogenital, and erythroid/immune systems, TRP channels are thought to contribute to diverse biological functions, which are not restricted to innocuous and noxious multimodal sensory transduction (heat, cold, touch, proprioception, pain, taste, etc.) but also involve cardiac function, gut motility, psychomotor activity, and cell survival, proliferation, and death. In addition, several specific mutations have been identified in the trp genes for some hereditary disorders $[12,14$ 17].

The expression of TRP proteins in the alimentary tract is not confined to sensory neurons. The repertoire includes the other major classes of cells constituting the tract such as epithelial, endothelial, and smooth muscle cells and has recently been extended to fibroblasts/myofibroblasts [13], which belong to a special category of cells tightly associated with colonal/intestinal remodeling with the ability to transform and replicate to produce various cytokines under inflammatory circumstances. For instance, calcitonin gene-related peptide and substance $P$ are known to be released by increased intracellular $\mathrm{Ca}^{2+}$ concentration through TRPV1 channel activation in sensory neurons $[18,19]$. It has been proposed that excessive expression of this channel may be causally related with the occurrence and/or progression of IBD [20, 21]. Moreover, a nonselective cation channel TRPC4, which can be activated by muscarinic Gprotein-coupled receptor stimulation, may be important for the excitatory control of intestinal smooth muscle cells [22-24]. Subsequent reports have implicated $\mathrm{Ca}^{2+}$ influx through TRPC4 channels in the initiation of spontaneous excitations in interstitial cells of Cajal, which regulate the gut automaticity [25]. More recently, we explored the potential roles of TRP channels in myofibroblastic $\mathrm{Ca}^{2+}$ signaling during intestinal inflammation and fibrosis. By using myofibroblast cell lines (CCD-18Co and InMyoFib) established from human colon epithelial and murine neonatal intestinal tissues, respectively, we could gain some key insights into the mechanisms underlying intestinal inflammatory and fibrotic remodeling processes [26].

In this chapter, we first describe the expression and function of TRPC channels in fibroblasts/ myofibroblasts and then briefly discuss their potential roles in gastrointestinal disorders. Since the tumor-transforming factor (tumor necrosis factor (TNF))- $\alpha$ has been shown to affect the expression level of TRPC1 protein and its associated $\mathrm{Ca}^{2+}$-transporting activity, the first part will be dedicated mainly to elucidating how TNF- $\alpha$ stimulates cyclooxygenase-2 (COX-2)dependent prostaglandin E2 (PGE2) production through the activation of TRPC1 channels and enhances $\mathrm{Ca}^{2+}$ dynamics in CCD-18Co myofibroblasts. We next clarify the impact of PGE2 production on myofibroblastic function, with particular interest in $\mathrm{Ca}^{2+}$-dependent regulation of transcription factors, that is, the nuclear factor of activated T-cell (NFAT) and the nuclear factor $\mathrm{kB}(\mathrm{NF}-\mathrm{kB})$. The results suggest that negative feedback regulation of PGE2 production in intestinal myofibroblasts through TRPC1-associated $\mathrm{Ca}^{2+}$ influx may be of significant clinical importance to protect the gut from exacerbation of inflammatory process and, thus, progression of IBD [27]. 
In the second part, we describe the functional implications of transforming growth factor $\beta 1$ (TGF- $\beta 1$ )-induced TRP channel activation in InMyoFib cells. Our studies so far suggest that TRP channels effectively regulate the expression of fibrosis-associated molecules and TGF- $\beta$ signaling in InMyoFib cells. Consistent with this, expressions of TRP channels and fibrosisassociated factors were found to be increased in the stenotic but not in non-stenotic regions of biopsy samples from CD patients' intestines, implying a therapeutic potential of targeting the channels [28]. From these advances, we further anticipate gaining a good clue to elucidating the complex interplay among commensal microbiota, intestinal cells, and the immune system of the gut, and how such interactions, with genetic susceptibility and modification by environmental factors, contribute to the pathogenesis of IBD.

\section{Roles of TRP proteins for the occurrence/progression of inflammatory bowel disease}

Consultation with the literature indicates that there is close correlation between IBD initiation/ progression and autoimmune abnormalities, which is characterized by aberrances in inflammatory responses of intestinal bacteria within the digestive tract. CD14-positive macrophages are markedly increased in the intestinal tract with CD pathology, where inflammatory cytokines including interleukin-6 and interleukin-23 (IL-6/IL-23) and TNF- $\alpha$ are excessively produced. The production of these cytokines, which can in turn activate adaptive immune reactions along with the production of IL-12 and IL-23, occurs at lower levels in the normal intestinal tract. However, suppressed immune responses of intestinal bacteria are inducible with higher production of IL-10, an anti-inflammatory cytokine involved in immune tolerance [29]. However, when chronic intestinal inflammation occurs, TNF- $\alpha$ or IL- 6 can be excessively produced, initiating an excessive inflammatory response. Originally, adaptive immune responses were considered to play the dominant role in the pathogenesis of IBD; however, novel immunological and genetic studies have demonstrated that innate immune responses are of comparable significance in inducing gut inflammation. Recent progress in understanding IBD pathogenesis sheds light on related disease mechanisms, including innate and adaptive immunities, and interactions between genetic influences and microbial or environmental factors [2].

TNF- $\alpha$ is central to inflammatory processes and acts as an endogenous tumor promoter [30]. Therapeutic antibodies against TNF- $\alpha$ exert dramatic ameliorating effects on inflammatory bowel syndrome; myofibroblasts have been found to play a key role in this disorder [31]. TNF$\alpha$ activates PGE2 production in myofibroblasts, fulfilling both protective and destructive roles in the gut. Although genetic deletion of the PGE2 receptor EP4 is detrimental to the gut, high concentrations of PGE2 analogs have also been shown to worsen clinical colitis (eventually leading to tumorigenesis), likely through the induction of pro-inflammatory reactions [3234]. The formation of PGE2 in myofibroblasts is primarily catalyzed by COX-2, which is expressed at low levels in unstimulated conditions before being rapidly induced in response to inflammatory cytokines, growth factors, and tumor promoters [35]. 
The myofibroblast cell line CCD-18Co expresses both COX forms and secretes PGE2, a feature that is significantly enhanced by TNF- $\alpha$ or IL-1 $\beta$ [30]. Evidence suggests that COX-2 expression and PGE2 production in myofibroblasts are controlled by intracellular $\mathrm{Ca}^{2+}$ concentration [36, 37]. However, the exact sources of $\mathrm{Ca}^{2+}$, which contribute to this process, remain entirely unclear. In general, there are two distinct sources of $\mathrm{Ca}^{2+}$ for elevating intracellular $\mathrm{Ca}^{2+}$ levels: $\mathrm{Ca}^{2+}$ influx across the plasma membrane and $\mathrm{Ca}^{2+}$ release from the endoplasmic reticulum (ER). $\mathrm{Ca}^{2+}$ influx can occur through voltage-gated $\mathrm{Ca}^{2+}$ channels, receptor-operated $\mathrm{Ca}^{2+}$-permeable channels (ROCs), and store-operated $\mathrm{Ca}^{2+}$ channels (SOCs). Recent studies have demonstrated that the canonical members of the TRP superfamily of proteins (TRPC) may contribute to SOC and ROC. The TRPC family consists of seven distinct isoforms designated as TRPC1-TRPC7 $[12,14,38,39]$. Presently, TRPC1 is regarded as one of the most plausible candidate molecules for SOC in many cell types [38, 39] and plays a critical role in intestinal epithelial restitution [40]. In some cell types, TRPC1 dynamically assembles with both stromal-interacting molecule 1 (STIM1) and Orai1 to generate a greater complexity in store-dependent $\mathrm{Ca}^{2+}$ influx mechanisms [41], although whether TRPC1 serves as a pore-forming SOC subunit still remains unclear.

In CCD-18Co cells, treatment with TNF- $\alpha$ greatly enhanced both $\mathrm{Ca}^{2+}$ influx induced by store depletion and cell-surface expression of TRPC1 protein and induced a cationic conductance. Selective inhibition of TRPC1 expression occurs by small interfering RNA or functionally effective TRPC1 antibody targeting the near-pore region of TRPC1 antagonized enhancement of store-dependent $\mathrm{Ca}^{2+}$ influx by TNF- $\alpha$, whereas TNF- $\alpha$ potentiated the induction of PGE2 production. Overexpression of TRPC1 in CCD-18Co produced opposite consequences [27]. We further elucidated that NF-kB and NFAT serve as important positive and negative transcriptional regulators, respectively, of TNF- $\alpha$-induced COX-2-dependent PGE2 production in colonic myofibroblasts, at the downstream of TRPC1-associated $\mathrm{Ca}^{2+}$ influx [27]. NFAT and $\mathrm{NF}-\mathrm{kB}$ are widely distributed $\mathrm{Ca}^{2+}$-dependent transcription factors capable of regulating a multitude of physiological and pathophysiological processes [42-44]. NFAT is activated through dephosphorylation by calcineurin, which is activated upon binding of $\mathrm{Ca}^{2+} / \mathrm{calmodu}^{-}$ lin. NFAT is reported to regulate COX-2 expression in colon carcinoma cells [45], and its activation can occur through $\mathrm{Ca}^{2+}$ influx associated with TRPC1-, TRPC3-, or TRPC6-associated SOC or ROC activities [46, 47]. The NF-kB transcription factor family plays a key role in several cellular functions (inflammation, apoptosis, cell survival, proliferation, angiogenesis, and innate and acquired immunity) as well as in regulating the expression of more than 500 different genes involved in inflammatory and immune responses [48, 49]. The anti-inflammatory natural compound curcumin acts as a principal mechanism to suppress the NF-kBmediated signaling, thereby modulating immune responses [50-52].

The fact that high doses of exogenous PGE2 analogs exacerbate clinical colitis in the TNBS model might be relevant to the use of misoprostol to prevent ulcers in patients who take antiarthritis medication. The side effects listed for misoprostol include a variety of gastrointestinal tract problems, and these deleterious actions of PGE2 are likely associated with the stimulation of the release of interleukin-23 from activated dendritic cells, which in turn facilitate the differentiation of helper $\mathrm{T}$ lymphocytes to the pro-inflammatory phenotype Th17. These 
opposing actions of PGE2 may imply that the extent of its production is crucial to determine the fate of intestinal mucosa, that is, the maintenance of integrity or disintegration. In this regard, the negative feedback regulation of PGE2 production in intestinal myofibroblasts through TRPC1-associated $\mathrm{Ca}^{2+}$ influx may be of significant clinical importance to protect the gut from exacerbation of inflammatory process and thus the progression of inflammatory bowel syndrome.

\section{Intestinal fibroblast/myofibroblast TRP channel and fibrosis}

Repeated cycles of inflammation and healing of the gut ultimately progress into intestinal fibrosis (Figure 1). Innate immune-signaling pathways are also important drivers of myofibroblast transdifferentiation, as they cause cellular activation and fibrosis. Numerous mediators, including PDGF, EGF, IGF-1 and -2, CTGF, IL-1, IL-13, stem cell factor, endothelins, angiotensin II, TGF- $\alpha$, TGF- $\beta$, bFGF, and peroxisome proliferator activator receptor- $\gamma$, promote myofibroblast proliferation and extracellular matrix (ECM) production. These activated myofibroblasts are central to fibrogenesis $[53,54]$.

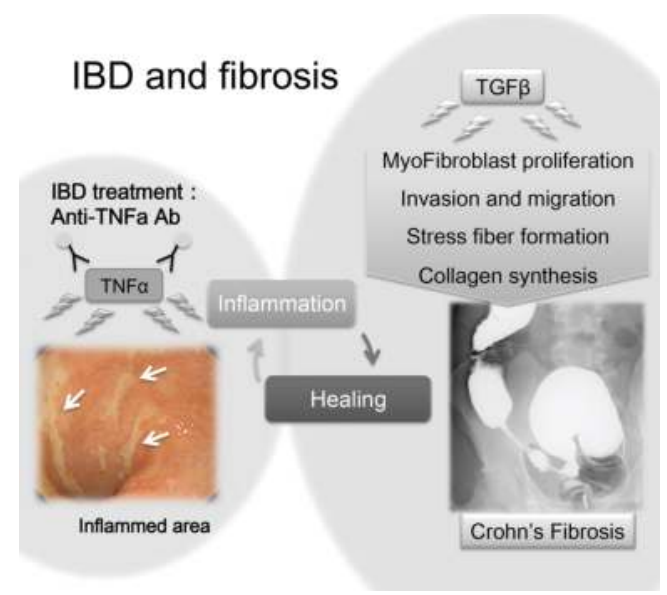

Figure 1. Inflammatory bowel disease and fibrosis. Repeated cycles of inflammation and healing of the gut ultimately progress into intestinal fibrosis. Endoscopic view of the inflamed area and a lower gastrointestinal series from a CD patient with fibrosis are shown. Colonoscopy and biopsy sampling showed a fibrotic lesion responsible for a colon stenosis.

TGF- $\beta$ is principal to the development of fibrotic stenosis in CD and in numerous cell types. TGF- $\beta$ secretion augments myofibroblast transformation. Canonical TGF- $\beta$ signaling commences with its binding to a TGF- $\beta$ type 2 receptor, which subsequently heterodimerizes with a TGF- $\beta$ type 1 receptor to form an active TGF- $\beta$ R 1 receptor complex. Activated TGF- $\beta$ type 1 receptor complex phosphorylates proteins against decapentaplegic homologs 2 and 3 (SMAD-2 and SMAD-3); activation of these transcription factors promotes collagen synthesis 
[55]. TGF- $\beta$ can also signal through noncanonical pathways involving extracellular signalregulated kinases (ERKs), c-Jun $\mathrm{N}$-terminal kinase, and p38-mitogen-activated protein kinase (p38-MAPK). Both canonical and noncanonical TGF- $\beta$-signaling pathways are implicated in myofibroblast cytokine production and fibrosis in the gut $[5,53]$. TGF- $\beta$ levels are elevated in the inflamed intestines of $\mathrm{CD}$ and ulcerative colitis patients, and abnormal TGF- $\beta$ signaling impairs intestinal immune tolerance and tissue repair [56]. In addition, TGF- $\beta$ receptortriggered-signaling cascades can be enhanced by calcineurin inhibitors cyclosporin $\mathrm{A}$ and FK506 [57, 58]. However, neutralizing TGF- $\beta 1$ in vivo as an anti-fibrotic approach in CD may be highly problematic, as this may actually lead to disease exacerbation, despite the potent anti-inflammatory and immunoregulatory properties of this cytokine. In addition to TGF- $\beta 1$, emerging evidence has shown that IL-13 and IL-17 are involved in intestinal fibrosis. IL-13 signaling via IL-13 receptor type 2 (IL-13R2) and subsequent TGF- $\beta 1$ production comprises the main fibrotic pathway in a model of chronic colitis [59]. IL-17A expression was found to be increased in the inflamed areas of patients with inflammatory bowel disease [60].

In response to tissue injury and profibrotic mediators including TGF- $\beta$ and PDGF, fibroblasts differentiate into myofibroblasts, and the activation and/or recruitment of fibroblasts with resistance to apoptosis result in fibrogenesis and subsequent fibrosis [61, 62]. It has been estimated that about $45 \%$ of human deaths are associated with fibroproliferative disorders including fibrosis [63]. Recently, anti-TNF- $\alpha$ antibodies were successfully introduced as antiinflammatory IBD therapies. However, for patients with fibrotic stenosis, there are only surgical treatments such as balloon dilation [64]. Approximately one-third of CD patients have severe intestinal strictures and obstructions (caused by excessive fibrosis) that are eventually fatal. In addition, treating CD patients with anti-TNF agents increases the risk of developing recurrent intestinal stenosis and sub-obstructive symptoms [65], necessitating repeated surgery [66]. In fact, many IBD patients are still suffering from re-stenosis of surgically treated regions, which greatly impairs the quality of life and can risk the lives of patients. Thus, there is an urgent need to establish alternative anti-fibrotic strategies to treat $C D$ patients and other individuals suffering from intestinal fibrotic complications beyond currently available antiinflammatory therapies. Unfortunately, little is currently known about intestinal woundhealing processes and pathogenic mechanisms by which chronic intestinal inflammation causes detrimental fibrosis, although a complex scenario involving numerous humoral factors has been suggested in experimental models [6-8].

Fibroblasts (vimentin+, $\alpha$-SMA-), located in the submucosal area of normal tissues, are central in maintaining structural formation, healing, and regeneration. Increased resident fibroblast populations are pivotal to fibrosis development. Fibroblasts isolated from IBD mucosa proliferate faster than normal, and this increase occurs after exposure to growth factors and pro-inflammatory cytokines, and after direct cell-to-cell contact with inflammatory cells. Fibroblast-to-myofibroblast (vimentin+, $\alpha$-SMA+) transformation plays a critical role in wound healing and tissue remodeling after injury $[8,67]$. Myofibroblasts synthesize ECM components and generate high contractile forces for wound retraction or tissue remodeling in developmental processes. However, persistent myofibroblast activity can underlie hypertrophic scarring, loss of tissue compliance, and even rampant fibrosis that is the basis for fibrotic 
disorders of the heart, skin, lung, kidney, skeletal muscle, and liver [6, 68, 69]. The myofibroblast is considered a hybrid cell type with both smooth muscle and fibroblast properties [8]. A defining feature of myofibroblast differentiation is the formation of $\alpha$-SMA stress fibers that provides a structural network for generating contractile forces [70]. Furthermore, intestinal stricture formation in CD is driven by the local excessive production of TGF- $\beta[5,71]$. It is well known that fibrosis is associated with excessive accumulation of ECM components, such as collagens, matrix metalloproteinases (MMPs), and tissue inhibitors of metalloproteinases (TIMPs) [63, 72, 73]. In addition, other ECM proteins, such as fibronectins, elastins, and fibrillins, are upregulated during the development of fibrosis. This is due mainly to increased synthesis and decreased degradation of ECM components. Notably, during this process, MMPs that degrade the ECM are upregulated, whereas TIMPs are downregulated [74].

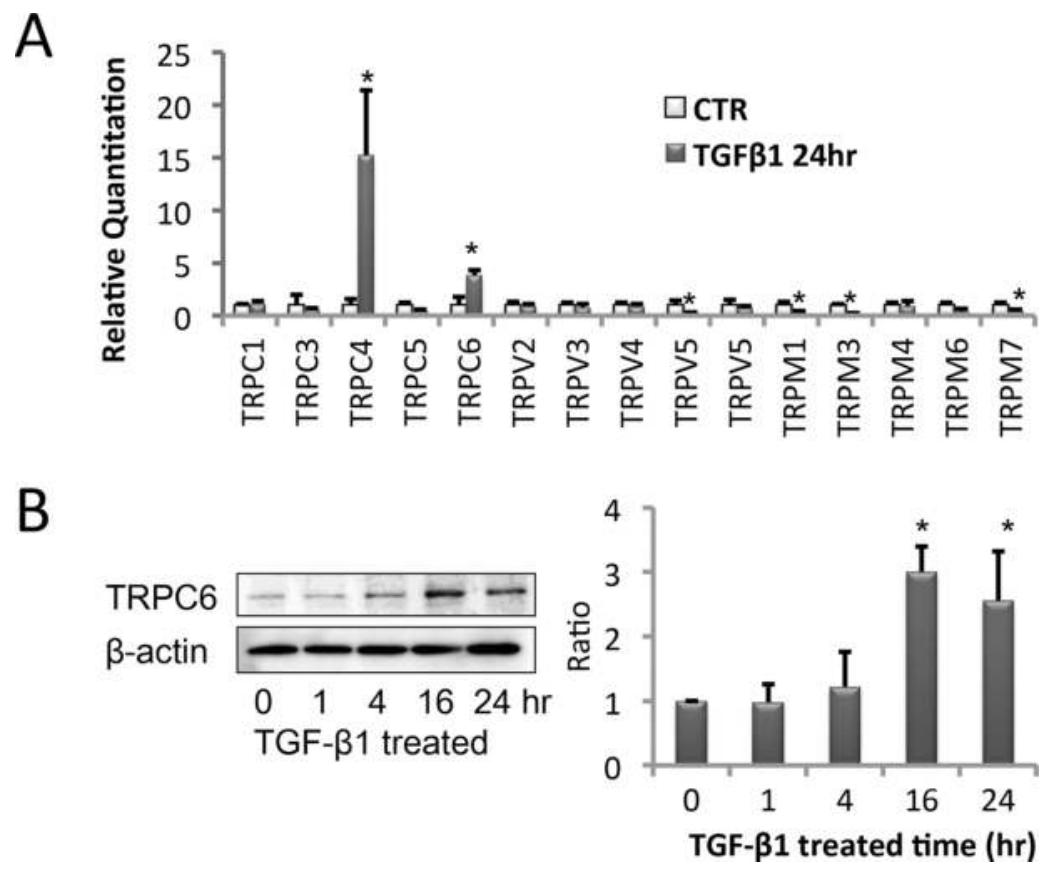

Figure 2. (A) TRP isoforms' mRNA in InMyoFibs. Results of real-time PCR analysis of the mRNA expression levels of TRPC1, $-C 3,-C 4,-C 5,-C 6,-V 2,-V 3,-V 4,-V 5,-V 6,-M 1,-M 3,-M 4,-M 6$, and $-M 7$ after treatment with TGF- $\beta 1$ (5 ng/mL, $24 \mathrm{~h}$ ) are shown. (B) Immunoblot data of time-dependent changes in TRPC6 protein expression (left panel). Data were normalized to an internal control $\left(\beta\right.$-actin) and are an average of four independent experiments (right panel). ${ }^{*} P<0.05$ compared with untreated cells $(n=4)$. This figure was modified from a figure in Ref. [28].

TRP channels are cellular sensors for a wide variety of physical and chemical stimuli [75-77]. For example, they are involved in the sensation of touch, smell, taste, temperature, and pain $[75,78-80]$. Recent studies have revealed that TRP channels also play essential roles in cell signaling and responses to innocuous or harmful environmental changes $[15,16,81]$. In addition, the activation of TRP channels changes the membrane potential, passes important 
signaling ions across the cell membrane, changes enzymatic activity, and initiates endocytosis or exocytosis $[12,75,82] . \mathrm{Ca}^{2+}$ is an essential signaling molecule implicated in various longterm cellular consequences, such as differentiation, gene expression, and cell proliferation, growth, and death, and it plays a significant role in regulating fibroblast functions [83-85]. TRPC channels are non-voltage-gated nonselective $\mathrm{Ca}^{2+}$-permeable channels. Enhanced $\mathrm{Ca}^{2+}$ influx has been implicated in both differentiation and cytoskeletal rearrangements of various cell types. Accumulating evidence suggests that fibrosis-associated events in myofibroblasts are controlled by intracellular $\mathrm{Ca}^{2+}$ concentration, which is mediated by some members of the TRP channel superfamily [14,86-88]. For example, TRPC1-mediated $\mathrm{Ca}^{2+}$ influx is essential for intestinal homeostasis/inflammation and progesterone-induced endometrial decidualization $[27,89] . \mathrm{Ca}^{2+}$ signaling through TRPM7 channels likely plays a key role in TGF- $\beta 1$-elicited fibrogenesis in human atrial fibroblasts [88]. Similarly, TRPC6/calcineurin-mediated signaling is essential for dermal and cardiac myofibroblast transformation, which occurs through complex interwoven pathways involving TGF- $\beta$, p38 mitogen-activated protein kinase, and serum response factor [70]. The formation of cell-to-cell contact is governed by $\mathrm{Ca}^{2+}$ signaling through TRPC4, which co-immunoprecipitates with junction proteins $\beta$-catenin and cadherin in vascular endothelial cells [90]. However, whether TRP channels play a role in intestinal fibrosis is not clearly understood.

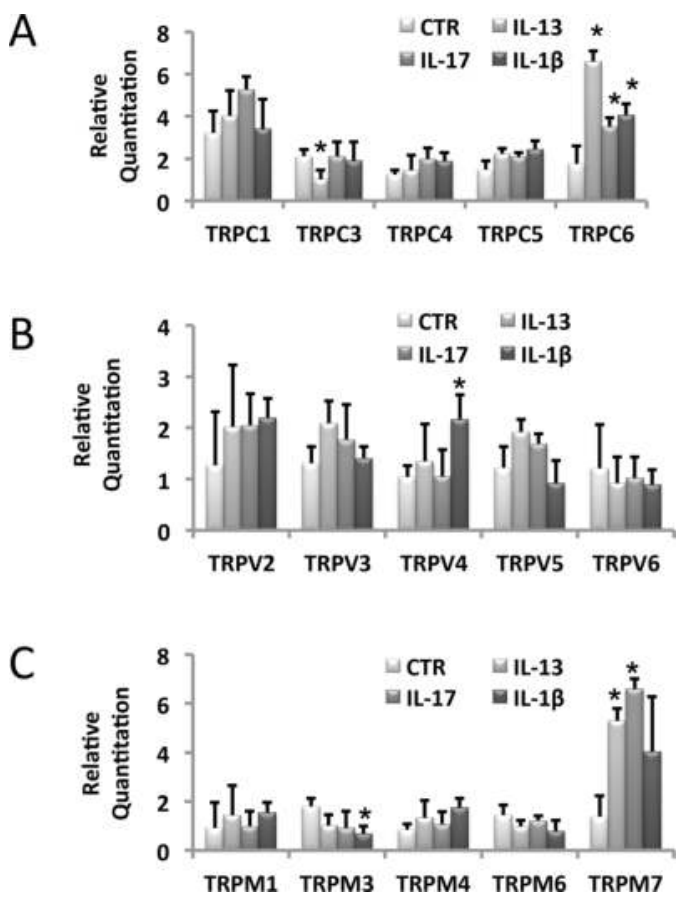

Figure 3. Real-time PCR analysis of TRPC1, $-C 3,-C 4,-C 5,-C 6,-V 2,-V 3,-V 4,-V 5,-V 6,-M 1,-M 3,-M 4,-M 6$, and $-M 7$ after a $24-\mathrm{h}$ treatment with IL-13 $(10 \mathrm{ng} / \mathrm{mL}), \mathrm{IL}-17(10 \mathrm{ng} / \mathrm{mL})$, and IL-1 $\beta(10 \mathrm{ng} / \mathrm{mL})$. This figure was modified from a supplementary figure in Ref. [28]. 
In intestinal myofibroblasts, not only TGF- $\beta 1$ but also IL-13 and IL-17 significantly upregulated TRPC6 expression (Figures 2 and 3). Myofibroblast TRPC6 is a key factor to modulate fibrosis through TGF signaling, and thus targeting TRPC6 may be a useful therapeutic regimen for CD patients with intestinal fibrosis [28]. The results showed that while increased TRPC6 activity promoted the TGF- $\beta 1$-mediated expression of $\alpha$-SMA and $\mathrm{N}$-cadherin and strengthened interactions between the three molecules, it also negatively regulated collagen synthesis and secretion of anti-fibrotic factors, such as IL-10 and IL-11 (ERK and p38-MAPK dependent) [9193]. Upregulated TRPC6 expression is essential for the formation of $\alpha$-SMA stress fibers and $\mathrm{N}$-cadherin-mediated adherens junctions, which, respectively, enable myofibroblasts to gain contractility and reinforce mutual intercellular connections [6, 94, 95]. Interestingly, adherens junctions appear in fibrotic tissues but are absent in normal tissues where fibroblasts do not develop the stress fibers [10]. These findings are consistent in part with a previous study that TRPC6-mediated $\mathrm{Ca}^{2+}$ influx was obligatory for myofibroblast differentiation in dermal and cardiac wound healing, although greater complexity appears to exist in the relationship between TRPC6-mediated signaling and intestinal fibrosis.

Furthermore, in our biopsy study, we examined samples from CD patients for the expression of TRPC4, TRPC6, $\alpha$-SMA, N-cadherin, cytokines, and ECM, and found that these molecules were all increased in TGF- $\beta 1$-treated InMyoFibs. The mRNA levels of TRPC6, ACTA2, CDH2, $I L-10, I L-11$, and COL1A1 were significantly higher in stenotic areas than in non-stenotic mucosal areas of CD patients, whereas that of TRPC4 was not significantly changed in 12 paired biopsy samples obtained from six patients (Figure 4). Stenotic lesions can be either inflammatory, fibrogenic, or neoplastic, or possess all of these characteristics. This means that therapeutic strategies distinguishing between these processes would yield improved outcomes compared with the currently available approaches. In this regard, more direct evidence that TRPC6 vitally contributes to the progression of excessive fibrosis in both an experimental model and in human tissues should help to elucidate the mechanism underlying the fibrotic process. This may be relevant not only to intestinal fibrosis but also to other fibrotic lesions of the skin, lung, and liver, where these channels are expressed at significant levels.

In addition to aforementioned mechanisms, the imbalance between MMP and TIMP, which maintain the state of remodeling and restitution, can accelerate structural changes of the bowel wall [1]. Microarray experiments showed that InMyoFib cells primarily express $M M P-1$, $M M P-2, T I M P-1$, and TIMP-2. When we next measured transcript expression of these molecules in stenotic areas from biopsy samples and TGF- $\beta 1$-treated cells, we found that their mRNA levels were significantly unregulated; however, TRPC6 siRNA pretreatment did not affect expression in TGF- $\beta 1$-treated cells.

The studies with intestinal fibroblast/myofibroblast propose a new proof of concept that TRPC6 may act as an anti-fibrotic mediator. The upregulation of this channel appears to inhibit the signaling cascades associated with intestinal fibrosis including SMAD-2 phosphorylation and myocardin expression, which in turn modulate collagen synthesis, actin fiber formation, and expression of $\mathrm{N}$-cadherin. Further evidence from biopsy samples suggests that the same mechanism may also operate in stenotic lesions of IBD. These results not only facilitate our 
understanding about this new role for TRPC6 in regulating fibrotic processes but also provide a novel molecular target for anti-fibrotic therapies to treat IBD in the future.
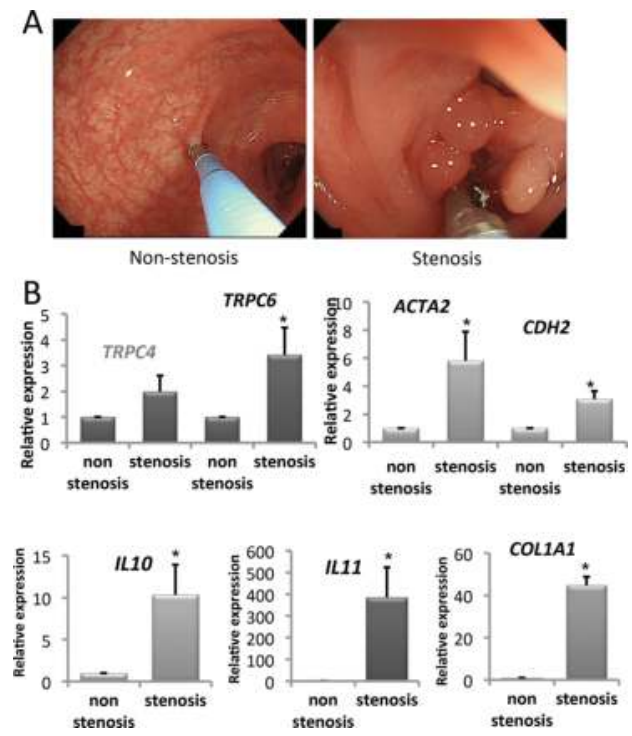

Figure 4. (A) Fibrosis in the colon: a clinical problem. Ulcerations and tissue damage are caused by chronic inflammation. This is followed by bowel wall fibrosis, leading to pseudopolyps or strictures reducing the colon. (B) Crohn's disease (CD) patient biopsies from non-stenotic and stenotic intestinal areas. The mRNA levels of TRPC4, TRPC6, ACTA2 ( $\alpha$-SMA), CDH2 (N-cadherin), IL-10, IL-11, and COL1A1 in biopsies were examined by real-time RT-PCR in non-stenotic and stenotic-inflamed mucosal tissues of CD patients. ${ }^{*} P<0.05$ versus non-stenotic samples (12 paired biopsy samples obtained from six patients). Figure 4B was modified from a figure in Ref. [28].

\section{Summary}

Several studies including our study have underscored the importance of intestinal fibroblast/ myofibroblast cells in IBD pathophysiology and epithelial barrier integrity, and accumulating evidence from preclinical and clinical studies has started to note an important contribution of TRP channels to many gastrointestinal remodeling processes. In this chapter, we summarized recent advances in this field, with particular emphasis on TNF- $\alpha$-activated TRPC1 and TGF$\beta$-activated TRPC6 expression and function in primary-cultured fibroblasts/myofibroblasts in the gastrointestinal tract, in conjunction with limited but interesting results from biopsy samples from CD patients. A noteworthy possibility from it is that the functionality of TRP channels may have unexpectedly tight correlation with inflammation- and fibrosis-associated processes in myofibroblasts in vitro and in vivo. Further investigation will be warranted to substantiate our yet-premature knowledge about this newly emerging field, which would hopefully lead to the exploitation of an unprecedentedly unique treatment for highly intractable inflammatory/fibrotic disorders with greatly compromised quality of life, such as IBD. 


\section{Acknowledgements}

This study was supported by the grants in aid to L.-H.K. from the Ministry of Education, Culture, Sports, Science, and Technology (Nos. 15K08978, 22790677, 25860571), a MEXTSupported Program funding research activities of female researchers, the Clinical Research Foundation, and the Central Research Institute of Fukuoka University.

\section{Author details}

Lin Hai Kurahara ${ }^{1^{*}}$, Keizo Hiraishi ${ }^{1}$, Kunihiko Aoyagi ${ }^{2}$, Yaopeng $\mathrm{Hu}^{1}$, Miho Sumiyoshi ${ }^{1}$ and Ryuji Inoue ${ }^{1}$

*Address all correspondence to: hailin@fukuoka-u.ac.jp

1 Department of Physiology, Fukuoka University School of Medicine, Fukuoka University, Fukuoka, Japan

2 Department of Gastroenterology, Fukuoka University School of Medicine, Fukuoka University, Fukuoka, Japan

\section{References}

[1] Karantanos, T. and Gazouli M., Inflammatory bowel disease: recent advances on genetics and innate immunity. Ann Gastroenterol, 2011. 24(3): p. 164-72.

[2] Zhang, Y.Z. and Li, Y.Y., Inflammatory bowel disease: pathogenesis. World J Gastroenterol, 2014. 20(1): p. 91-9.

[3] Meier, J.K., et al., Specific differences in migratory function of myofibroblasts isolated from Crohn's disease fistulae and strictures. Inflamm Bowel Dis, 2011. 17(1): p. 202-12.

[4] Valentich, J.D., et al., Phenotypic characterization of an intestinal subepithelial myofibroblast cell line. Am J Physiol, 1997. 272(5 Pt 1): p. C1513-24.

[5] Biancheri, P., et al., The role of transforming growth factor (TGF)-beta in modulating the immune response and fibrogenesis in the gut. Cytokine Growth Factor Rev, 2014. 25(1): p. 45-55.

[6] Hinz, B. and Gabbiani, G., Fibrosis: recent advances in myofibroblast biology and new therapeutic perspectives. F1000 Biol Rep, 2010. 2: p. 78.

[7] Latella, G., et al., Can we prevent, reduce or reverse intestinal fibrosis in IBD? Eur Rev Med Pharmacol Sci, 2013. 17(10): p. 1283-304. 
[8] Hinz, B., Formation and function of the myofibroblast during tissue repair. J Invest Dermatol, 2007. 127(3): p. 526-37.

[9] Hourigan, L.F., et al., Fibrosis in chronic hepatitis C correlates significantly with body mass index and steatosis. Hepatology, 1999. 29(4): p. 1215-9.

[10] Hinz, B., et al., Myofibroblast development is characterized by specific cell-cell adherens junctions. Mol Biol Cell, 2004. 15(9): p. 4310-20.

[11] Hinz, B., et al., The myofibroblast: one function, multiple origins. Am J Pathol, 2007. 170(6): p. 1807-16.

[12] Flockerzi, V., An introduction on TRP channels. Handb Exp Pharmacol, 2007. 179: p. 119.

[13] Phillips, A.M., Bull, A., and Kelly, L.E., Identification of a Drosophila gene encoding a calmodulin-binding protein with homology to the trp phototransduction gene. Neuron, 1992. 8(4): p. 631-42.

[14] Inoue, R., et al., Transient receptor potential channels in cardiovascular function and disease. Circ Res, 2006. 99(2): p. 119-31.

[15] Inoue, R., Jian, Z., and Kawarabayashi, Y., Mechanosensitive TRP channels in cardiovascular pathophysiology. Pharmacol Ther, 2009. 123(3): p. 371-85.

[16] Inoue, R., et al., Regulation of cardiovascular TRP channel functions along the NOcGMP-PKG axis. Expert Rev Clin Pharmacol, 2010. 3(3): p. 347-60.

[17] Nilius, B., TRP channels in disease. Biochim Biophys Acta, 2007. 1772(8): p. 805-12.

[18] Szallasi, A. and Blumberg, P.M., Complex regulation of TRPV1 by vanilloids, in TRP Ion Channel Function in Sensory Transduction and Cellular Signaling Cascades, Liedtke, W.B. and Heller, S., Editors. Boca Raton, FL; CRC Press/Taylor \& Francis 2007.

[19] Szallasi, A., et al., The vanilloid receptor TRPV1: 10 years from channel cloning to antagonist proof-of-concept. Nat Rev Drug Discov, 2007. 6(5): p. 357-72.

[20] Yiangou, Y., et al., ATP-gated ion channel P2X(3) is increased in human inflammatory bowel disease. Neurogastroenterol Motil, 2001. 13(4): p. 365-9.

[21] Yiangou, Y., et al., Increased acid-sensing ion channel ASIC-3 in inflamed human intestine. Eur J Gastroenterol Hepatol, 2001. 13(8): p. 891-6.

[22] Inoue, R., TRP channels as a newly emerging non-voltage-gated CA2+ entry channel superfamily. Curr Pharm Des, 2005. 11(15): p. 1899-914.

[23] Kuriyama, H., et al., Physiological features of visceral smooth muscle cells, with special reference to receptors and ion channels. Physiol Rev, 1998. 78(3): p. 811-920.

[24] Insuk, S.O., et al., Molecular basis and characteristics of KATP channel in human corporal smooth muscle cells. Int J Impot Res, 2003. 15(4): p. 258-66. 
[25] Torihashi, S., et al., Calcium oscillation linked to pacemaking of interstitial cells of Cajal: requirement of calcium influx and localization of TRP4 in caveolae. J Biol Chem, 2002. 277(21): p. 19191-7.

[26] Yu, X., et al., TRP channel functions in the gastrointestinal tract. Semin Immunopathol, 2016 May;38(3):385-96.

[27] Hai, L., et al., Counteracting effect of TRPC1-associated Ca2+ influx on TNFalpha-induced COX-2-dependent prostaglandin E2 production in human colonic myofibroblasts. Am J Physiol Gastrointest Liver Physiol, 2011. 301(2): p. G35667.

[28] Kurahara, L.H., et al., Intestinal myofibroblast TRPC6 channel may contribute to stenotic fibrosis in Crohn's disease. Inflamm Bowel Dis, 2015. 21(3): p. 496-506.

[29] Neurath, M.F., Cytokines in inflammatory bowel disease. Nat Rev Immunol, 2014. 14(5): p. 329-42.

[30] Kim, E.C., et al., Cytokine-mediated PGE2 expression in human colonic fibroblasts. Am J Physiol, 1998. 275(4 Pt 1): p. C988-94.

[31] Di Sabatino, A., et al., Functional modulation of Crohn's disease myofibroblasts by antitumor necrosis factor antibodies. Gastroenterology, 2007. 133(1): p. 137-49.

[32] Kabashima, K., et al., The prostaglandin receptor EP4 suppresses colitis, mucosal damage and CD4 cell activation in the gut. J Clin Invest, 2002. 109(7): p. 88393.

[33] Sheibanie, A.F., et al., The proinflammatory effect of prostaglandin E2 in experimental inflammatory bowel disease is mediated through the IL-23-->IL-17 axis. J Immunol, 2007. 178(12): p. $8138-47$.

[34] Sugimoto, Y. and Narumiya, S., Prostaglandin E receptors. J Biol Chem, 2007. 282(16): p. 11613-7.

[35] Smith, W.L., DeWitt, D.L., and Garavito, R.M., Cyclooxygenases: structural, cellular, and molecular biology. Annu Rev Biochem, 2000. 69: p. 145-82.

[36] Ogata, S., et al., Ca2+ stimulates COX-2 expression through calcium-sensing receptor in fibroblasts. Biochem Biophys Res Commun, 2006. 351(4): p. 808-14.

[37] Zhu, Y., et al., Ca2+- and PKC-dependent stimulation of PGE2 synthesis by deoxycholic acid in human colonic fibroblasts. Am J Physiol Gastrointest Liver Physiol, 2002. 283(3): p. G503-10.

[38] Ambudkar, I.S., et al., TRPC1: the link between functionally distinct store-operated calcium channels. Cell Calcium, 2007. 42(2): p. 213-23.

[39] Putney, J.W., Physiological mechanisms of TRPC activation. Pflugers Arch, 2005. 451(1): p. 29-34. 
[40] Rao, J.N., et al., TRPC1 functions as a store-operated Ca2+ channel in intestinal epithelial cells and regulates early mucosal restitution after wounding. Am J Physiol Gastrointest Liver Physiol, 2006. 290(4): p. G782-92.

[41] Ong, H.L., et al., Dynamic assembly of TRPC1-STIM1-Orai1 ternary complex is involved in store-operated calcium influx. Evidence for similarities in store-operated and calcium release-activated calcium channel components. J Biol Chem, 2007. 282(12): p. 9105-16.

[42] Crabtree, G.R. and Olson, E.N., NFAT signaling: choreographing the social lives of cells. Cell, 2002. 109(Suppl.): p. S67-79.

[43] Timmerman, L.A., et al., Rapid shuttling of NF-AT in discrimination of Ca2+ signals and immunosuppression. Nature, 1996. 383(6603): p. 837-40.

[44] Yates, L.L. and Gorecki, D.C., The nuclear factor-kappaB (NF-kappaB): from a versatile transcription factor to a ubiquitous therapeutic target. Acta Biochim Pol, 2006. 53(4): p. 651-62.

[45] Duque, J., Fresno, M., and Iniguez, M.A., Expression and function of the nuclear factor of activated T cells in colon carcinoma cells: involvement in the regulation of cyclooxygenase-2. J Biol Chem, 2005. 280(10): p. 8686-93.

[46] Ohba, T., et al., Upregulation of TRPC1 in the development of cardiac hypertrophy. J Mol Cell Cardiol, 2007. 42(3): p. 498-507.

[47] Thebault, S., et al., Differential role of transient receptor potential channels in Ca2+entry and proliferation of prostate cancer epithelial cells. Cancer Res, 2006. 66(4): p. 2038-47.

[48] Basak, S., et al., A fourth IkappaB protein within the NF-kappaB signaling module. Cell, 2007. 128(2): p. 369-81.

[49] Karin, M. and Greten, F.R., NF-kappaB: linking inflammation and immunity to cancer development and progression. Nat Rev Immunol, 2005. 5(10): p. 749-59.

[50] Vecchi Brumatti, L., et al., Curcumin and inflammatory bowel disease: potential and limits of innovative treatments. Molecules, 2014. 19(12): p. 21127-53.

[51] Holt, P.R., Katz, S., and Kirshoff, R., Curcumin therapy in inflammatory bowel disease: a pilot study. Dig Dis Sci, 2005. 50(11): p. 2191-3.

[52] Holt, P.R., Curcumin for inflammatory bowel disease: a caution. Clin Gastroenterol Hepatol, 2016. 14(1): p. 168.

[53] Leask, A., Potential therapeutic targets for cardiac fibrosis: TGFbeta, angiotensin, endothelin, CCN2, and PDGF, partners in fibroblast activation. Circ Res, 2010. 106(11): p. $1675-80$.

[54] Derynck, R. and Zhang, Y.E., Smad-dependent and Smad-independent pathways in TGF-beta family signalling. Nature, 2003. 425(6958): p. 577-84. 
[55] Medina, C., et al., Transforming growth factor-beta type 1 receptor (ALK5) and Smad proteins mediate TIMP-1 and collagen synthesis in experimental intestinal fibrosis. J Pathol, 2011. 224(4): p. 461-72.

[56] Babyatsky, M.W., Rossiter, G., and Podolsky, D.K., Expression of transforming growth factors alpha and beta in colonic mucosa in inflammatory bowel disease. Gastroenterology, 1996. 110(4): p. 975-84.

[57] Akool el, S., et al., Molecular mechanisms of TGF beta receptor-triggered signaling cascades rapidly induced by the calcineurin inhibitors cyclosporin A and FK506. J Immunol, 2008. 181(4): p. 2831-45.

[58] Chiasson, V.L., et al., Endothelial cell transforming growth factor-beta receptor activation causes tacrolimus-induced renal arteriolar hyalinosis. Kidney Int, 2012. 82(8): p. 857-66.

[59] Fichtner-Feigl, S., et al., IL-13 signaling via IL-13R alpha2 induces major downstream fibrogenic factors mediating fibrosis in chronic TNBS colitis. Gastroenterology, 2008. 135(6): p. 2003-13.

[60] Biancheri, P., et al., The role of interleukin 17 in Crohn's disease-associated intestinal fibrosis. Fibrogenesis Tissue Repair, 2013. 6(1): p. 13.

[61] Arribillaga, L., et al., Therapeutic effect of a peptide inhibitor of TGF-beta on pulmonary fibrosis. Cytokine, 2011. 53(3): p. 327-33.

[62] Biernacka, A., Dobaczewski, M., and Frangogiannis, N.G., TGF-beta signaling in fibrosis. Growth Factors, 2011. 29(5): p. 196-202.

[63] Ghosh, A.K. and Vaughan, D.E., Fibrosis: is it a coactivator disease? Front Biosci (Elite Ed), 2012. 4: p. 1556-70.

[64] Bettenworth, D. and Rieder, F., Medical therapy of stricturing Crohn's disease: what the gut can learn from other organs - a systematic review. Fibrogenesis Tissue Repair, 2014. 7(1): p. 5.

[65] Condino, G., et al., Anti-TNF-alpha treatments and obstructive symptoms in Crohn's disease: a prospective study. Dig Liver Dis, 2013. 45(3): p. 258-62.

[66] Rieder, F., et al., Wound healing and fibrosis in intestinal disease. Gut, 2007. 56(1): p. 130-9.

[67] Tomasek, J.J., et al., Myofibroblasts and mechano-regulation of connective tissue remodelling. Nat Rev Mol Cell Biol, 2002. 3(5): p. 349-63.

[68] Wynn, T.A., Cellular and molecular mechanisms of fibrosis. J Pathol, 2008. 214(2): p. 199-210.

[69] Wang, Y.S., et al., Role of miR-145 in cardiac myofibroblast differentiation. J Mol Cell Cardiol, 2014. 66: p. 94-105. 
[70] Davis, J., et al., A TRPC6-dependent pathway for myofibroblast transdifferentiation and wound healing in vivo. Dev Cell, 2012. 23(4): p. 705-15.

[71] Di Sabatino, A., et al., Transforming growth factor beta signalling and matrix metalloproteinases in the mucosa overlying Crohn's disease strictures. Gut, 2009. 58(6): p. 77789.

[72] Muro, A.F., et al., An essential role for fibronectin extra type III domain A in pulmonary fibrosis. Am J Respir Crit Care Med, 2008. 177(6): p. 638-45.

[73] Zeisberg, M. and Kalluri, R., Cellular mechanisms of tissue fibrosis. 1. Common and organ-specific mechanisms associated with tissue fibrosis. Am J Physiol Cell Physiol, 2013. 304(3): p. C216-25.

[74] Hemmann, S., et al., Expression of MMPs and TIMPs in liver fibrosis - a systematic review with special emphasis on anti-fibrotic strategies. J Hepatol, 2007. 46(5): p. 95575.

[75] Zheng, J., Molecular mechanism of TRP channels. Compr Physiol, 2013. 3(1): p. 221-42.

[76] Inoue, R., Ito, Y., and Mori, Y., TRP-related proteins as new target molecules: their correspondence to native receptor-operated cation channels. Tanpakushitsu Kakusan Koso, 2000. 45(6 Suppl): p. 1038-46.

[77] Inoue, R., Ito, Y., and Mori, Y., The TRP proteins, a rapidly expanding Ca2+ entry channel family and a new molecular target for drug development. Nihon Rinsho, 2002. 60(1): p. 18-24.

[78] Clapham, D.E., Runnels, L.W., and Strubing, C., The TRP ion channel family. Nat Rev Neurosci, 2001. 2(6): p. 387-96.

[79] Clapham, D.E., TRP channels as cellular sensors. Nature, 2003. 426(6966): p. 517-24.

[80] Ramsey, I.S., Delling, M., and Clapham, D.E., An introduction to TRP channels. Annu Rev Physiol, 2006. 68: p. 619-47.

[81] Cheng, Y. and Nash, H.A., Drosophila TRP channels require a protein with a distinctive motif encoded by the inaF locus. Proc Natl Acad Sci USA, 2007. 104(45): p. 17730-4.

[82] Xu, T., et al., Novel insights into TRPM7 function in fibrotic diseases: a potential therapeutic target. J Cell Physiol, 2015. 230(6): p. 1163-9.

[83] Berridge, M.J., Calcium signalling remodelling and disease. Biochem Soc Trans, 2012. 40: p. 297-309.

[84] Yue, L.X., Xie, J., and Nattel, S., Molecular determinants of cardiac fibroblast electrical function and therapeutic implications for atrial fibrillation. Cardiovas Res, 2011. 89(4): p. 744-753.

[85] Saliba, Y., et al., Evidence of a role for fibroblast transient receptor potential canonical 3 Ca2+ channel in renal fibrosis. J Am Soc Nephrol, 2015. 26(8): p. 1855-1876. 
[86] Nishida, M., et al., Galpha12/13-mediated up-regulation of TRPC6 negatively regulates endothelin-1-induced cardiac myofibroblast formation and collagen synthesis through nuclear factor of activated T cells activation. J Biol Chem, 2007. 282(32): p. 23117-28.

[87] Onohara, N., et al., TRPC3 and TRPC6 are essential for angiotensin II-induced cardiac hypertrophy. EMBO J, 2006. 25(22): p. 5305-16.

[88] Du, J., et al., TRPM7-mediated Ca2+ signals confer fibrogenesis in human atrial fibrillation. Circ Res, 2010. 106(5): p. 992-1003.

[89] Kawarabayashi, Y., et al., Critical role of TRPC1-mediated $\mathrm{Ca}(2)(+)$ entry in decidualization of human endometrial stromal cells. Mol Endocrinol, 2012. 26(5): p. 846-58.

[90] Graziani, A., et al., Cell-cell contact formation governs Ca2+ signaling by TRPC4 in the vascular endothelium: evidence for a regulatory TRPC4-beta-catenin interaction. J Biol Chem, 2010. 285(6): p. 4213-23.

[91] Aoki, H., et al., Autocrine loop between TGF-beta1 and IL-1beta through Smad3- and ERK-dependent pathways in rat pancreatic stellate cells. Am J Physiol Cell Physiol, 2006. 290(4): p. C1100-8.

[92] Saraiva, M. and O'Garra, A., The regulation of IL-10 production by immune cells. Nat Rev Immunol, 2010. 10(3): p. 170-81.

[93] Kido, S., et al., Mechanical stress activates Smad pathway through PKCdelta to enhance interleukin-11 gene transcription in osteoblasts. PLoS One, 5(9): e744-e744 (2010).

[94] Ina, K., et al., Significance of alpha-SMA in myofibroblasts emerging in renal tubulointerstitial fibrosis. Histol Histopathol, 2011. 26(7): p. 855-66.

[95] Speca, S., et al., Cellular and molecular mechanisms of intestinal fibrosis. World J Gastroenterol, 2012. 18(28): p. 3635-61. 